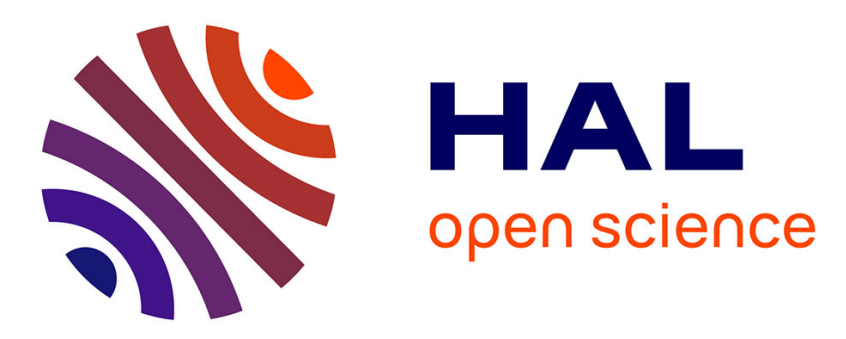

\title{
Separation of Coulomb fission from nuclear fission at medium energies
}

\author{
A. Bonaccorso, Z. Zelazny, E. Piasecki
}

\section{To cite this version:}

A. Bonaccorso, Z. Zelazny, E. Piasecki. Separation of Coulomb fission from nuclear fission at medium energies . Zeitschrift für Physik A, 1997, 358 (3), pp.329-336. 10.1007/s002180050336 . in2p301593751

\section{HAL Id: in2p3-01593751 https://hal.in2p3.fr/in2p3-01593751}

Submitted on 26 Sep 2017

HAL is a multi-disciplinary open access archive for the deposit and dissemination of scientific research documents, whether they are published or not. The documents may come from teaching and research institutions in France or abroad, or from public or private research centers.
L'archive ouverte pluridisciplinaire HAL, est destinée au dépôt et à la diffusion de documents scientifiques de niveau recherche, publiés ou non, émanant des établissements d'enseignement et de recherche français ou étrangers, des laboratoires publics ou privés. 

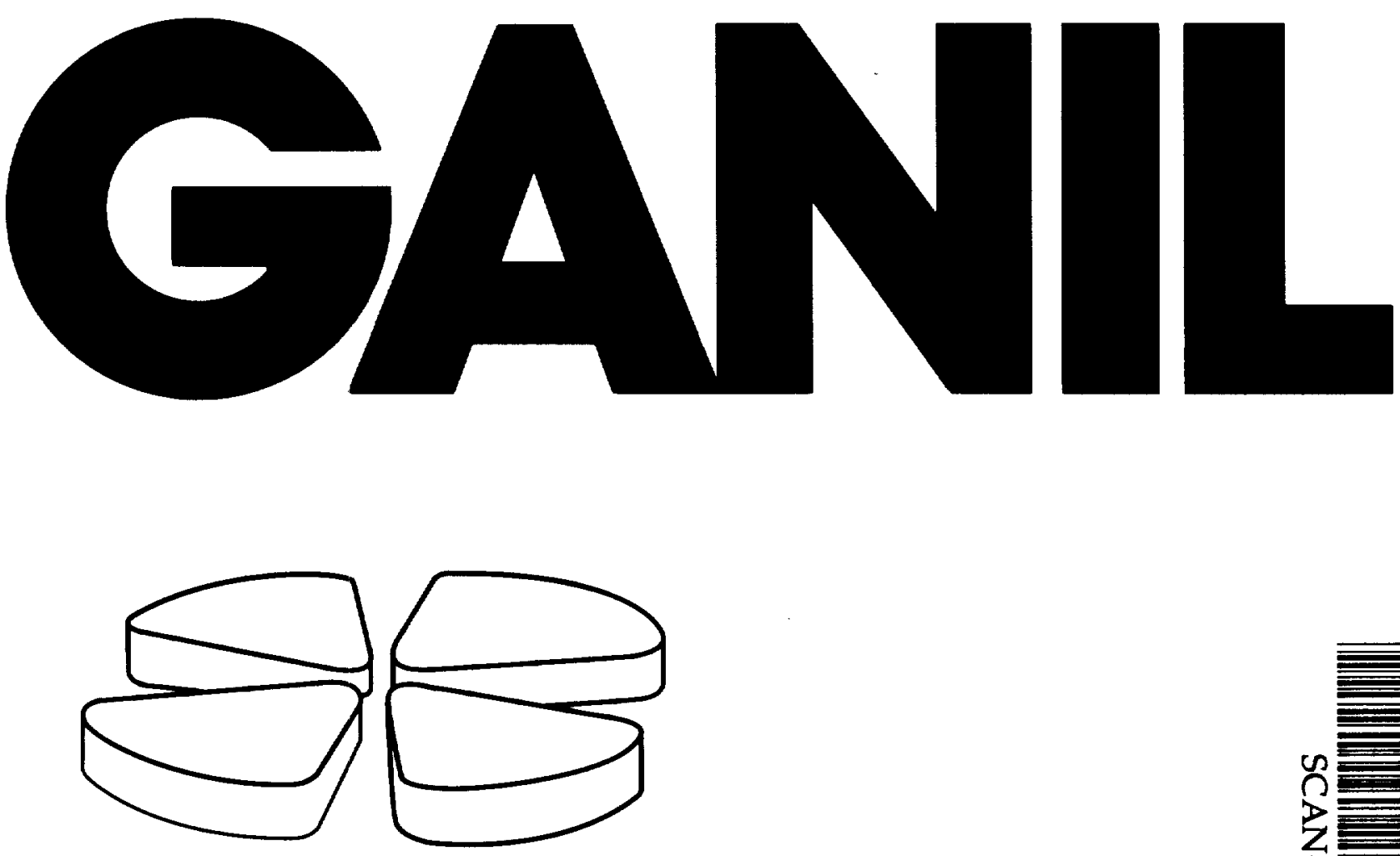

Separation of Coulomb fission from nuclear fission at medium energies

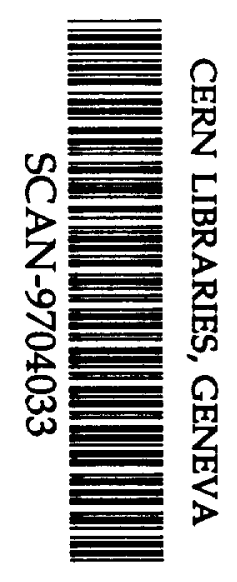
A. Bonaccorso,
Z. Żelazny;
E. Piasecki ${ }^{\dagger}$
52114

*Istituto Nazionale di Fisica Nucleare, Sezione di Pisa, 56100 Pisa, Italy

Institute of Experimental Physics, Warsaw University, Hoża 69, 00-681 Warsaw, Poland and GANIL, 14021 Caen, France

\section{Accepted for publication in Zeitschrift für Physik A}




\title{
Separation of Coulomb fission from nuclear fission at medium energies
}

\author{
A. Bonaccorso, $\quad$ Z. Żelazny ${ }^{\dagger} \quad$ E. Piasecki ${ }^{\dagger}$ \\ *Istituto Nazionale di Fisica Nucleare, Sezione di Pisa, 56100 Pisa, Italy \\ †Institute of Experimental Physics, Warsaw University, Hoża 69, 00-681 Warsaw, \\ Poland and GANIL, 14021 Caen, France
}

\begin{abstract}
The cross sections for fission induced by one neutron transfer and by the electromagnetic field in the reaction $24.3 \mathrm{MeV} / \mathrm{u}{ }^{238} \mathrm{U}+{ }^{197} \mathrm{Au}$ are calculated and compared to the experimental data. It turns up that the two calculated cross sections differ by five orders of magnitude at a distance of closest approach of $25 \mathrm{fm}$. It is shown that in the experiment in which one is able to select the events corresponding to a large distance of closest approach, a separation of Coulomb fission from nuclear fission events can be efficiently obtained.
\end{abstract}

\section{Introduction}

The Coulomb fission (CF) reaction, i.e. fission of a nucleus induced by the time-varying Coulomb field of another nucleus passing by in the vicinity, possibly possess some unique (although never unambiguously observed so far) characteristics, i.e. a presumed time scale faster by four orders of magnitude than the time scale usually observed in fission [1]. This phenomenon, which should not be confounded with the so called "prompt fission" [2], might be caused by bypassing the compound nucleus stage due to the direct coupling of the electric field to the collective motion. There have been quite a few papers devoted to experimental studies of CF ([3] - [16]), unfortunately, only 
some of them can be considered convincing. The main reason for this is the background problem caused by nuclear interaction, which plagues the studies of the phenomenon.

Because of the lack of clear experimental signature of $\mathrm{CF}$, there have been essentially two ways of increasing the signal/background ratio: minimizing the nuclear interaction by using the subbarrier projectile energies (Refs. [3] [10]) or maximizing the electromagnetic interaction by using the relativistic heavy ion projectiles (see [11]-[16]).

In the first approach one looked for fission after back-scattering (head-on collisions) between two heavy nuclei. Using the deeply subbarrier projectile one certainly decreases the probability of nuclear interaction (at the expense of the total cross section). However even then there were serious doubts whether the process was really induced by the electromagnetic field alone. E.g. the authors of Ref.[4] stated that their data published in Ref. [3] "did not show unambiguously that there was any pure Coulomb fission although there were some positive indications", thus in the next paper they tried to resolve this question. The conclusion of this paper was that "it seems more likely that the observed events are due to a nuclear process such as transfer induced fission".Also the authors of Ref.[5] explained the observed fission events from reactions of ${ }^{16} \mathrm{O}$ and ${ }^{19} \mathrm{~F}$ with ${ }^{232} \mathrm{Th}$ at sub-Coulomb $(0.85-1.0) \mathrm{V}_{C B}$ energy range as a result of the combination of Coulomb excitation and particle transfer reactions, where $\mathrm{V}_{C B}$ denotes the Coulomb barrier. The conclusion of another relevant paper, where the interaction of $\mathrm{Xe}$ ions with ${ }^{238} \mathrm{U}$ at energies of (0.7-1.0) $\mathrm{V}_{C B}$ was studied (Ref.[6]) was that the authors could not decide whether the fission cross section below $\sim 0.9 \mathrm{~V}_{C B}$ was due to sequential fission fission after direct nuclear reactions or rather to a dominant Coulomb fission. On the other hand the authors of Ref.[18] came to conclusion that for the reaction ${ }^{238} \mathrm{U}+{ }^{238} \mathrm{U}$ one should go down to the energy of $0.82 \mathrm{~V}_{C B}$ to be sure that more than $90 \%$ of the measured fission cross section was Coulomb fission. The reason of the difficulties is that, as it was shown in a series of papers [17]-[20], the one-neutron transfer is observed up to extremely large distances of closest approach of the collision partners, e.g. equal to $24 \mathrm{fm}$ in the ${ }^{238} \mathrm{U}+{ }^{238} \mathrm{U}$ reaction and $27 \mathrm{fm}$ in the ${ }^{238} \mathrm{U}+{ }^{197} \mathrm{Au}$ reaction. Only checking that the back-scattered nuclei are the same as the projectile ones, enabled [8] convincing identification of $\mathrm{CF}$, although even there one could not distinguish between inelastic Coulomb and inelastic nuclear excitation. These experimental difficulties caused (with the exception of an unpublished work [10]), that the obtained data were limited essentially to cross sections, 
which for fission of ${ }^{232} \mathrm{Th},{ }^{238} \mathrm{U}$ and ${ }^{248} \mathrm{Cm}$ were in the $1-10 \mathrm{mb}$ range. For the excellent review of the subbarrier CF results, the reader is referred to Ref.[1].

Another experimental approach made use of the fact that the CF cross section strongly rises with projectile energy, reaching few barns for $E_{\text {proj }}$ of the order of $1 \mathrm{GeV} / \mathrm{u}$. Unfortunately, the nuclear fission (NF) cross section is comparable at these energies what makes the distinction between the two processes even more difficult. To decompose the experimental distributions into the CF and NF contributions, the authors of paper[12] utilized the information from the folding- and azimuthal-angle and velocity distributions and applied an elaborate simulation. In other papers ([11],[13]-[16]) the authors subtracted (after an appropriate scaling) the fission fragment charge distributions from reactions on light and heavy targets. However, since in experiments of this type, no specific means have been applied to discriminate event-by-event CF from NF, these studies of CF have also been limited to cross section determination. Clearly, the event-by-event distinction between $\mathrm{CF}$ and NF is necessary if one wants to investigate the CF properties, especially if one is interested in studying the differences between these two processes.

In the paper [21] a new approach was used. The energy was intermediate between subbarrier and relativistic and the measurement of angular distribution of fissioning ${ }^{238} \mathrm{U}$ nuclei was performed with the aim of looking for the events corresponding to large values of the distance of closest approach (D), beyond the range of nuclear interaction. The heavy system and sufficiently high projectile energy $\left(24.3 \mathrm{MeV} / \mathrm{u}{ }^{238} \mathrm{U}+{ }^{197} \mathrm{Au}\right)$ resulted in a large value of the Sommerfeld parameter $(n \approx 230)$ thus allowing use of the classical notion of trajectory. This enabled controlling $D$ by means of the scattering angle variable reconstructed for each event from registered fission fragments. One should remember, however, that the small scattering angle in itself is not a sufficient criterion for the identification of peripheral process: for very dissipative collisions the attractive nuclear field bends the projectile trajectory towards small or even negative angles. To distinguish between these two situations yet another variable has been measured simultaneously: the multiplicity of neutrons accompanying each fission event. Thus the signature used in Ref. [21] for event-by-event selection of CF was: small scattering angle (corresponding to the $\mathrm{D} \geq 25 \mathrm{fm}$ ) and small neutron multiplicity.

On the other hand, from a theoretical point of view semiclassical methods based on the notion of a classical trajectory for the description of the ion-ion 
relative motion have been widely used, with great success, for the description of peripheral reactions both below [22] and above the Coulomb barrier [23][27]. The notion of 'average classical trajectory' has also found support from the comparison of full quantum mechanical calculations and semiclassical calculations [28].

The aim of the present paper is to calculate the cross sections, their dependence on the distance of closest approach and the excitation energies for $\mathrm{CF}$ and NF induced by one neutron transfer and to compare them with the experimental data published in [21].

\section{The one-neutron transfer calculations}

As it is known experimentally[19], at large values of $D$ the one-neutron transfer to ${ }^{238} \mathrm{U}$ is the dominant transfer reaction. It is also known that in such reactions the donor remains essentially cold: all the excitation energy goes to the acceptor [29],[30]. Since we are interested in fission, with a barrier of the order of $6 \mathrm{MeV}$, the possibility of ${ }^{237} \mathrm{U}$ production in a stripping reaction of ${ }^{238} \mathrm{U}$ is less troublesome as a source of fission background than the pick-up giving rise to the excited ${ }^{239} \mathrm{U}$. Thus the numerical results of calculations given below pertain the latter case although the formalism is a general one.

As we have mentioned above for the subbarrier energies the one neutron transfer has been observed for very large D. The essential mechanism is a tunnelling process of a neutron through the potential barrier, as it was proposed already by Breit et al. [31]. At higher energies it has been shown in Refs. [32] - [33] and in the series of papers by D.M.Brink and collaborators $[23]$ - [27] that the transfer mechanism is still the same and that the transfer probability decays exponentially with the distance of closest approach with a parameter $\eta$ which is some kind of average momentum between the initial and final state. The actual form of $\eta$ will be discussed in the following.

In this paper we follow the Brink et al. formalism and calculate the one neutron transfer cross section in the reaction ${ }^{197} \mathrm{Au}\left({ }^{238} \mathrm{U},{ }^{239} \mathrm{U}\right){ }^{196} \mathrm{Au}$. We start from the semiclassical transfer cross section given by [34]

$$
\left.\frac{d \sigma}{d \Omega}\right|_{D}=\left.\left(\frac{d \sigma}{d \Omega}\right)_{c l}\right|_{D} P_{t r} P_{e l}
$$

and we assume the strong absorption model for $\mathrm{P}_{e l}$, the elastic nucleusnucleus scattering probability, such that $\mathrm{P}_{e l}=1$ if $D \geq R_{s}$ and $P_{e l}=0$ if 
$D<R_{s}$. D is the distance of closest approach of the Coulomb trajectory of relative motion. The strong absorption radius is taken as

$$
R_{s}=1.4\left(A_{P}^{1 / 3}+A_{T}^{1 / 3}\right)[f m] .
$$

The transfer probability $\mathrm{P}_{t r}$ is given by Eq. (3.15) of [24] for transfer between bound states and by Eqs.(2.1) and (2.2) of [27] for transfer to a final continuum state.

In both cases $P_{t r}$ was calculated analytically and the main dependence on the distance of closest approach $\mathrm{D}$ was found to be

$$
P_{t r} \sim e^{-2 \eta D}
$$

where $\eta^{2}=-2 m \tilde{\varepsilon} / \hbar^{2}$ and

$$
\tilde{\varepsilon}=\frac{1}{2}\left(\varepsilon_{i}+\varepsilon_{f}\right)-\frac{1}{4}\left(\frac{\left(\varepsilon_{i}-\varepsilon_{f}\right)}{\frac{1}{2} m v^{2}}+\frac{1}{2} m v^{2}\right) .
$$

Here, $\varepsilon_{i}$ is the neutron initial separation energy in the donor. In the case of final bound state, $\varepsilon_{f}=S_{n}$ is just the neutron separation energy in the acceptor + neutron system. In the case of final continuum states and if there is no donor excitation, $\varepsilon_{f}$ is related to the acceptor excitation energy by $E^{*}=\varepsilon_{f}+S_{n}$. The quantity $\frac{1}{2} m v^{2}$ is the neutron incident energy at distance $\mathrm{D}, m$ is the neutron mass, $v=\sqrt{2\left(E_{C M}-\frac{Z_{p} Z_{\mathcal{T}} e^{2}}{D}\right) / \mu}$ and $\mu$ is the projectile-target reduced mass.

The initial and final neutron binding energies used in our calculations of transfer to the ground state together with the asymptotic normalization constants for the initial and final wave functions (cf.[24]) are given in the Table.

For the continuum calculations the initial state parameters are still the same while the S-matrix, which describes the neutron-target final state interaction and which enters in the final continuum state definition, has been calculated using the following optical model parameters which have been obtained from Ref. [35]

$$
\begin{gathered}
V_{R}=45.45-0.22 \varepsilon_{f} \mathrm{MeV} \quad r_{R}=1.265 \mathrm{fm} \quad a_{R}=0.55 \mathrm{fm} \\
W_{D}=2.2+0.47 \varepsilon_{f} \mathrm{MeV} \quad r_{i}=1.235 \mathrm{fm} \quad a_{i}=0.3 \mathrm{fm} \\
V_{S O}=9 \mathrm{MeV} \quad W_{S O}=5.5 \mathrm{MeV} \quad r_{S O}=1.25 \mathrm{fm} \quad a_{S O}=0.55 \mathrm{fm}
\end{gathered}
$$


We have calculated $d \sigma / d D$ starting from Eq. (1), assuming that the $\left(\frac{d \sigma}{d \Omega}\right)_{c l}$ is given by the Rutherford cross section and using the classical relation between the deflection angle (in the CMS) and the distance of closest approach

$$
k D=n\left(1+\sqrt{1+\operatorname{cotg}^{2} \frac{\theta}{2}}\right),
$$

where $n=Z_{P} Z_{T} \mathrm{e}^{2} / \hbar V$ and $k=\mu V / \hbar$ are the Sommerfeld parameter and wave number of relative motion, respectively. $V$ is the asymptotic relative velocity of the target and projectile.

In our reaction the Sommerfeld parameter is quite large $n=233$ and the system should behave classically because the diffraction effects are expected to be important only if $n \approx 1$. According to Landau and Lifshitz [36] this is possible provided the conditions $\Delta D<<D, \Delta \theta<<\theta$ and $\Delta \theta>>1 /(k b)$ are satisfied. Here $b \simeq D-a_{c}$ is the impact parameter (cf. Eq.(2.4) of [24]) and $a_{c}=n / k$ is the Coulomb length parameter.

In our case the wavelength of the relative motion at the strong interaction distance $R_{s}=16.8 \mathrm{fm}$ equals $\lambda=8 \cdot 10^{-3} \mathrm{fm}$, thus being much smaller than the distance itself. The $R_{s}$ is the smallest characteristic distance relevant in peripheral reactions discussed here. In fact at the distances of closest approach $\mathrm{D} \geq 25 \mathrm{fm}$ considered in this paper the wavelength is even smaller. Thus the first condition is fulfilled.

The other two conditions are important to ensure that the correspondence angle-distance of closest approach be applied in the region of small angles for which diffraction effects are however not important. The experimental angular resolution of the analyzed data was $\Delta \theta \sim 0.8^{\circ}$, and the data correspond to the angular region $\theta=2.5^{\circ}-4.5^{\circ}$, therefore the second condition is readily fulfilled. The last condition requires $\Delta \theta>>0.033^{\circ}$ if $D=17 \mathrm{fm}$ and $\Delta \theta>>0.021^{\circ}$ if $D=25 \mathrm{fm}$, which is also satisfied by the experimental conditions.

The calculated $d \sigma / d D$ for $1 \mathrm{n}$ pick-up from ${ }^{197} \mathrm{Au}$ to the ground state of ${ }^{239} \mathrm{U}$ for different energies of ${ }^{238} \mathrm{U}$ are shown in Fig.1. One can see the strong decrease of the $1 \mathrm{n}$ transfer probability with $\mathrm{D}$ as well as with the projectile energy. In the same figure we present the results of similar calculations for transfer to continuum, which in contrast to the former case can lead to fission, giving rise to the background in the CF studies. It is interesting to notice that at $E_{\text {proj }}=8.1 \mathrm{MeV} / u$ both transfer to the ground state and to the continuum results fall on the same line. This is a threshold effect due 
to the fact that at this incident energy the matching conditions [25] provide that the neutron final energy should be very close to zero. It shows that our normalization for the final bound and continuum final states are consistent. Then there is a smooth transition between the two cases and the absolute values of the transfer cross sections are reliable.

The dependence on projectile energy of the 1n transfer cross section integrated over $D>25 \mathrm{fm}$ is presented in the inset of this figure. One can see that after an initial rise due to the opening of the continuum channels, the cross section saturates and then begins to decrease slowly. This is due to the fact that we show the inclusive cross section, which is the sum of the neutron absorption and elastic breakup cross sections [26]. Increasing the incident energy the absorption decreases while the elastic breakup increases and both phenomena contribute to the acceptor excitation energy spectrum. Since in the experiment [21] the mass number of the fissioning nuclei was not determined well enough to distinguish between these two phenomena, then for the purpose of this paper it is appropriate to calculate the inclusive cross section.

The transfer to unbound states generates the wide distribution of excitation energy of the acceptor $U$ nucleus. The excitation energy distributions of the ${ }^{239} \mathrm{U}$ nuclei produced in this process for projectile energy of $24.3 \mathrm{MeV}$ (as in Ref. [21]) depend to some extent on D. However, as Fig.2 shows, the most probable excitation is slightly lower than the projectile incident energy per nucleon, in agreement with optimum kinematical matching conditions [25]. The consequences of this for interpreting the experiment [21] will be discussed at the end of the next section. Here we just want to mention that various experiments might sample different parts of the excitation energy distribution. For example a transfer to the continuum experiment provides information on the whole excitation energy spectrum of the acceptor while a fission experiment like Ref.[21] can give information only on the excitation energy spectrum above the fission barrier. Because of this in Fig. 3 we compare the results of calculations for transfer to the continuum for three ranges of ${ }^{239} \mathrm{U}$ excitation energy. The value $6.8 \mathrm{MeV}$ equals the fission barrier of this nucleus. The range of the excitation energy [5.8 - 11.8] MeV results in a mean value of $10 \mathrm{MeV}$. This is the value relevant to the experiment [21] since the measured fragment charge distribution enabled to show that the events classified as CF correspond to fission of cold nuclei: the nuclei in which the upper limit of excitation energy was estimated to be $10 \mathrm{MeV}$. Finally, the range $[5.8-34.8] \mathrm{MeV}$ covers all the excitation energy possibly generated 
in ${ }^{239} \mathrm{U}$ by $1 \mathrm{n}$ pick-up from ${ }^{197} \mathrm{Au}$ in a scattering process characterized by a distance of closest approach larger than $25 \mathrm{fm}$. Comparing these results to those of Fig.1 we can see that the transfer to the ground state is in these conditions much less probable.

\section{The Coulomb fission calculations}

In order to compare experimental results of Coulomb fission cross sections from [21] with the theoretical predictions, we performed a calculation within the Bertulani and Baur relativistic model [37] of electromagnetic dissociation with fission chosen as the exit channel. This approach, originally proposed by Fermi [38] and later on developed by Weizsacker [39] and Williams [40, 41], replaces the electromagnetic field of the interacting projectile and target with the equivalent photon flux.

The probability for the occurrence of an electromagnetic fission in a nuclear collision is given by the following formula [37]:

$$
P_{e m f}(b)=\int N(\omega, b) \sigma_{\gamma, f}(\omega) d \omega / \omega
$$

where $\sigma_{\gamma, f}$ is the photofission cross section for the photon energy $\hbar \omega$, given as the product of the fission probability $P_{f}(\omega)$ by the electromagnetic cross section $\sigma_{e m}$ and $N(\omega, b)$ is the number of equivalent photons incident on the target per unit area. The integral runs over all the frequency $\omega$ spectrum of the virtual radiation.

The total electromagnetic fission cross section is calculated integrating the probability shown above over the impact parameter from a minimum value $b_{\min }=R_{s}$ (the smallest impact parameter with still no influence of nuclear interaction) to $b=\infty$ :

$$
\sigma_{e m f}=2 \pi \int_{R_{s}}^{\infty} P_{e m f}(b) b d b=\int n(\omega) \sigma_{\gamma, f}(\omega) \frac{d \omega}{\omega},
$$

where $n(\omega)=2 \pi \int_{R_{s}}^{\infty} N(\omega, b) b d b$.

Making use of the multipole expansion of the interaction the electromagnetic cross section can be written in the following form:

$$
\sigma_{e m}=\sum_{l} \int\left\{n_{E l}(\omega) \sigma^{E l}(\omega)+n_{M l}(\omega) \sigma^{M l}(\omega)\right\} \frac{d \omega}{\omega}
$$


The $n_{E l}(\omega)$ and $n_{M l}(\omega)$ are called the virtual photon spectra for the relevant electric and magnetic multipolarities, respectively.

From the experimental papers dealing with uranium fission [42]-[44] one can conclude that the only excitation multipolarities which is necessary to include are E1 and E2 and thus the Coulomb fission cross section reads :

$$
\sigma_{e m f}=\int n_{E 1}(\omega) \sigma^{E 1}(\omega) P_{f}(\omega) \frac{d \omega}{\omega}+\int n_{E 2}(\omega) \sigma^{E 2}(\omega) P_{f}(\omega) \frac{d \omega}{\omega}
$$

where $\sigma^{E 1}$ and $\sigma^{E 2}$ are photoabsorption cross sections for GDR and GQR, respectively. The fission probability $\mathrm{P}_{f}(\omega)$ for ${ }^{238} \mathrm{U}$ was experimentally determined in [43] and [44]. From just above threshold $(\sim 5.8 \mathrm{MeV})$ to $\sim 12.3 \mathrm{MeV}$ it is nearly constant with a value 0.22 . Around $12.3 \mathrm{MeV}$ the second chance fission switches on (the $(\gamma, \mathrm{nf})$ reaction) and the fission probability rises sharply to a value of about 0.42 [44]. To parametrize these data we have applied the prescription proposed by Aumann et al. [45].

The E1 photoabsorption cross section was assumed to be of the double Lorentzian form and the corresponding parameters were taken from [43]. The strength, width and resonance energy for the first and the second Lorentzian were equal to $301 \mathrm{mb}, 2.90 \mathrm{MeV}$ and $10.96 \mathrm{MeV}$ and to $369 \mathrm{mb}, 4.53 \mathrm{MeV}$ and $14.04 \mathrm{MeV}$, respectively [43]. The isoscalar and isovector components of the E2 photoabsorption cross section were accounted for. Both of them were assumed to be of the single Lorentzian form. The strength, width and the resonance energy of the isoscalar quadrupole (equal to $6.3 \mathrm{mb}, 3.0 \mathrm{MeV}$ and $9.9 \mathrm{MeV}$, respectively) were taken from [46] and [47], while the corresponding parameters of the isovector quadrupole (equal to $20.0 \mathrm{mb}, 5.0 \mathrm{MeV}$ and $21.6 \mathrm{MeV}$, respectively) were adopted from [46] and [48].

The virtual photon spectra $n_{E 1}(\omega)$ and $n_{E 2}(\omega)$ were calculated according to the prescriptions given by Bertulani and Baur [37]. Since in Ref. [21] the experimental CF cross section was estimated for $D \geq 25 \mathrm{fm}$, in our calculations a value of $b_{\min }=23 \mathrm{fm}$ is used, which corresponds to $\mathrm{D}=25 \mathrm{fm}$. However, at our projectile energy we have to take into account the simplification of the formalism. Namely, one assumes a straight line trajectory of the scattered projectile. Alder and Winther [49] suggested an approximate way to account for the effect of Rutherford bending. We followed their suggestion which in fact rescaled the straight line $b_{\min }$ value with a new value bigger by about $3 \mathrm{fm}$ for the considered projectile-target system and projectile energy. Figure 4 shows in log scale the equivalent photon spectra and the photoab- 
sorption cross sections for both multipolarities E1 and E2. It is seen that both photon spectra fall down very steeply with the increasing photon energy (by about 3 orders of magnitude per $5 \mathrm{MeV}$ ). In the interesting energy region (i.e. $5-15 \mathrm{MeV}$ ) the quadrupole spectrum dominates over the dipole one by a factor of one hundred. On the other hand the photoabsorption cross sections reveal a contrary relation: the dipole cross section is more than hundred times bigger than the quadrupole one. In such circumstances these two multipolarities give comparable contributions to the total electromagnetic fission cross section.

Fission probability and its threshold character play an important role in this calculation, particularly as the photon spectra decrease so rapidly with energy. The net result of this interplay is presented in Fig. 5, where the products of fission probability, photoabsorption cross section and the virtual photon spectra are plotted for both multipolarities. We can see three curves peaked at the barrier energy, which integrated over the photon energy give us the final results of our calculation. The dipole contribution $(1.3 \mathrm{mb})$ is not more than about $30 \%$ of the total Coulomb fission cross section ( $\sigma^{E 1}$ $+\sigma^{E 2 s}+\sigma^{E 2 v}$ ) which amounts to $4.5 \mathrm{mb}$. The quadrupole isovector part is of minor importance being equal to $0.4 \mathrm{mb}$, while the isoscalar amounts to $2.8 \mathrm{mb}$. The main source of uncertainty of this calculation (about $30 \%$ ) is due to lack of detailed information about the subbarrier part of $\mathrm{P}_{f}$. This can give rise to relatively large error because of the exponential shape of virtual photon spectra (cf. Fig. 4). The values of the cross section given above are probably overestimated by $10-20$ per cent because of the approximate bending correction [50]. The projectile energy dependence of the $\sigma^{E 1}$, $\sigma^{E 2 s}, \sigma^{E 2 v}$ and their sum (for D $\geq 25 \mathrm{fm}$ ) is presented in Fig. 6. One can see that increasing the projectile energy to $50 \mathrm{MeV} / \mathrm{u}$ would result in a ten-fold amplification of the Coulomb fission cross section.

As we mentioned above, the formulae of Bertulani and Baur [37] are integrated over the impact parameter. As in this paper we are mainly interested in the impact parameter dependence of the electromagnetic fission cross section (the experimental data of this type are reported in [21]) we differentiated numerically the results of the Bertulani and Baur formulae. The outcome of this calculation is presented in Fig. 7, where it is compared with the corresponding value for fission after $1 \mathrm{n}$ transfer giving rise to ${ }^{239} \mathrm{U}$ excited in the interval of $[5.8-11.8] \mathrm{MeV}$ (see sect. 2). 


\section{Comparison with experimental data}

In Ref. [21] the experimental cross section for CF was estimated (for D $\geq 25 \mathrm{fm}$ ) to be of the order of $1 \mathrm{mb}$. This value is in agreement with the calculations described in the previous section and it is much higher than the theoretical cross section for fission-after-transfer reaction. One can see in Fig. 7 that under the conditions of this experiment (relatively high energy) and for sufficiently large $\mathrm{D}$ the $\mathrm{CF}$ and transfer-fission cross sections differ by five or more orders of magnitude, therefore the background of fission following in transfer is there negligible.

The cross sections for NF and CF differ strongly not only in the order of magnitude but also in their dependence on the distance of closest approach. This is illustrated in Fig. 8, where - in order to evidentiate the different slopes - all the curves shown as well as the deconvoluted experimental data are arbitrarily normalized to the same value at $D=18 \mathrm{fm}$. One can see that the slopes of curves corresponding to $\mathrm{CF}$ and NF processes are quite different, this being a consequence of the difference in range between the nuclear and electromagnetic interactions. The calculated transfer-fission cross section has a slope very similar to that observed experimentally in subbarrier in transfer reaction [20]. The dependence of the deconvoluted experimental data is instead very close to that of the CF calculated here, especially for $D>19 \mathrm{fm}$. Whether the slightly larger slope, seen for the smaller distance, is a real effect, caused by some admixture of nuclear component or it is an experimental artifact, it could be decided only by performing a new experiment with better angular resolution than that of Ref.[21]. One should remember that $D=18 \mathrm{fm}$ corresponds to the deflection angle of $6.5 \mathrm{deg}$ (in the LAB), which is very close to the grazing angle $(7.3 \mathrm{deg})$, the difference being within the experimental resolution of $0.9 \mathrm{deg}$.

We discuss now briefly the possibility that the data we are concerned with in this paper could contain contribution of nuclear fission following nuclear inelastic excitations.

According to [22] at high incident energies ( $>20 \mathrm{MeV} / u$ ) grazing collisions could excite the giant resonances. However microscopic calculations of the form factors containing both nuclear and Coulomb contributions, show that at distances larger than the sum of projectile and target radii, the form factors start to follow the behaviour characteristic of Coulomb excitations. For example in Ref.[51] it is shown that in the scattering of heavy systems like ${ }^{40} \mathrm{Ar}+{ }^{238} \mathrm{U}$ at $E_{\text {proj }}=41 \mathrm{MeV} / \mathrm{u}$ nuclear excitations are completely neg- 
ligible for $\theta_{C M} \leq 6^{\circ}$, which using Eq.(8) gives $D \geq 17.1 \mathrm{fm}$, a value larger by $3.4 \mathrm{fm}$ than the strong absorption radius one can obtain from Eq.(2). For our system instead $R_{s}=16.8 \mathrm{fm}$, thus for $D>20.5 \mathrm{fm}$ nuclear excitations should be already negligible. We remind the reader that in this paper we are considering experimental data corresponding to $D>25 \mathrm{fm}$.

Furthermore, in Chap.V of [22] it is also shown that the one nucleon transfer form factor has a much slower decay at large distances than the inelastic form factor. Therefore having already ensured that the fission induced by one neutron transfer gives a negligible contribution to the data discussed in this paper, we can a fortiori exclude the possibility of a significant nuclear inelastic excitation induced fission.

Another argument for the interpretation of the events observed in the experiment [21], as coming from the CF, concerns the excitation energy. In this experiment the observed fission fragment charge distribution was used as an internal thermometer and it was estimated that the mean excitation energy of fissioning nuclei was lower than $10 \mathrm{MeV}$. We have shown in Fig. 3 that after $1 \mathrm{n}$ transfer the ${ }^{239} \mathrm{U}$ excitation energy spectrum is peaked at about $20 \mathrm{MeV}$. On the other hand the peak excitation energy generated by the electromagnetic interaction should be in these conditions much lower because of the steepness of the virtual photon spectrum (cf. Fig. 5), in agreement with experimental results.

\section{Summary and conclusions}

In this paper we have calculated the cross sections for fission of uranium induced by $1 n$ transfer and by the electromagnetic field in the reaction 24.3 $\mathrm{MeV} / \mathrm{u}{ }^{238} \mathrm{U}+{ }^{197} \mathrm{Au}$ and we have compared the outcomes of the calculations to the experimental findings of Ref.[21]. Special emphasis has been put on the dependence of the calculated quantities on the distance of closest approach.

It appears that for the system considered in this paper the calculated cross sections corresponding to the two processes differ very much: at $D \geq 25 \mathrm{fm}$ the CF is by 5 orders of magnitude more probable than the transfer-fission reaction. Therefore the contamination of the CF by $1 n$ transfer seems to be of minor importance. Especially the use of relatively high projectile energy is very effective in reducing the 1 n transfer background for sufficiently large distance of closest approach. Further increase of the projectile energy (from 24 to $50 \mathrm{MeV} / \mathrm{u}$ ) would result in a ten-fold amplification of the Coulomb fission 
cross section without any substantial increase of the in transfer probability.

The calculated CF cross section is comparable to the experimental one [21]. In these experimental conditions, the contributions of dipole and isoscalar quadrupole excitations are comparable, while the isovector quadrupole excitation gives an almost negligible contribution.

The slope of $\mathrm{d} \sigma / \mathrm{dD}$ vs. $\mathrm{D}$ for $1 \mathrm{n}$ transfer is much steeper than that calculated for $\mathrm{CF}$, the latter being very close to the experimental one.

In the conditions of this experiment, the calculations suggest a strong heating of ${ }^{239} \mathrm{U}$ following the process of neutron pick-up, contrary to expectations for Coulomb fission. Since the experimental charge distribution of fragments [21] shows the characteristics of fission of cold nuclei, this is yet another indication of a good separation of CF from NF events in the experiment in which one selects the events coming from sufficiently large distances of closest approach.

\section{Acknowledgments}

The authors would like to express their gratitude to Dr. Joel Galin for his continuous interest in the subject and for numerous and stimulating discussions, to Prof. D.M. Brink and Dr.A.Vitturi for some useful suggestions and to Prof. W. von Oertzen for a critical reading of the manuscript. Part of this work was done while two of the authors (E.P. and Z.Z.) were visiting Grand Accelerateur National d'Tons Lourds (Caen, France). They are pleased to acknowledge the financial support of the IN2P3-Poland Convention and the warm hospitality they enjoyed during their stay .

\section{References}

[1] Oberacker, V.E., Pinkston, W.T., Kruse, H.G.W., Rep. Prog. Phys. 48, 327 (1985)

[2] Kruse, K., Pinkston, W.T., Greiner, W., Oberacker, V., J. Phys. G: Nucl. Phys. 5, L105 (1979) 
[3] Colombani, P., Butler, P.A., Lee, I.Y., Cline, D., Diamond, R.M., Stephens, F.S., Ward, D., Phys. Lett. B65, 39 (1976)

[4] Butler, P.A., Lee, I.Y., Newton, J.O., El-Masri, Y., Aleonard, M.M., Colombani, P., Diamond, R.M., Stephens, F.S., Lougheed, R.W., Hulet, E.K., Phys. Lett. B68, 122 (1977)

[5] Leigh, J.R., Diamond, R.M., Johnston, A., Newton, J.O., Sie, S.H., Phys.Rev.Lett. 42,153 (1979)

[6] Franz, G., Kratz, J.V., Brüchle, W., Folger, H., Haefner, B., Z. Phys.A291, 167 (1979)

[7] Habs, D., Metag, V., Schukraft, J., Specht, H.J., Wene, C.O., Hildenbrand, K.D., Z. Phys. A283, 261 (1977)

[8] Backe, H., Weik, F., Butler, P.A., Metag, V., Wilhelmy, J.B., Habs, D., Himmele, G., Specht, H.J., Phys. Rev. Lett. 43, 1077 (1979)

[9] Himmele, G., Backe, H., Butler, P.A., Habs, D., Metag, V., Wilhelmy, J.B., Specht, H.J., Nucl.Phys. A391, 191 (1982)

[10] Schukraft, J.G., Ph.D. Thesis, University of Heidelberg, 1983, unpublished

[11] Justice, M.L., Blumenfeld, Y., Colonna, N., Delis, D.N., Guarino, G., Hanold, K., Meng, J.C., Peaslee, G.F., Wozniak, G.J., Moretto, L.G., Phys.Rev. C49, R5 (1994)

[12] Polikanov, S., Brüchle, W., Folger, H., Jäger, E., Krogulski, T., Schädel, M., Schimpf, E., Wirth, G., Aumann, T., Kratz, J.V., Stiel, E., Trautmann, N., Z.Phys. A350, 221 (1994)

[13] Schmidt, K.-H., Heinz, A., Clerc, H.-G., Blank, B., Brohm, T., Czajkowski, S., Donzaud, C., Geissel, H., Hanelt, E., Irnich, H., Itkis, M.C., de Jong, M., Junghans, A., Magel, A., Münzenberg, G., Nickel, F., Pfützner, M., Piechaczek, A., Röhl, C., Scheidenberger, C., Schwab, W., Steinhäuser, S., Sümmerer, K., Trinder, W., Voss, B., Zhdanov, S.V., Phys.Lett. B325, 313 (1994) 
[14] Aumann, T., Brüchle, W., Fauerbach, M., Hill, J.C., Kratz, J.V., Schädel, M., Stiel., E., Sümmerer, K., Wirth, G., Nucl.Phys. A569, 157c (1994)

[15] Schmidt, K.-H., Heinz, A., Geissel, H., Münzenberg, G., Schwab, W., Sümmerer, K., Clerc, H.-G., Brohm, T., Grewe, A., Hanelt, E., de Jong, M., Junghans, A., Müller, J., Röhl, C., Steinhäuser, S., Voss, B., Andriamonje, S., Blank, B., Czajkowski, S., Praoff, M.S., Itkis, M.G., Ignatyuk, A.V., Kudyaev, G.A., Pfützner, M., Zhdanov, S., Proceedings of the European Physical Society (XV Nuclear Physics Divisional Conference), St Petersburg, April 1995, Editors Yu.Oganessian, R.Kalpakchieva, W von Oertzen World Scientific, pag.222.

[16] Rubehn, Th., Müller, W.F.J., Bassini, R., Begemann-Blaich, M., Blaich, Th., Ferrero, A., Groß, C., Imme, G., Iori, I., Kunde, G.J., Kunze, W.D., Lindenstruth, V., Lynen, U., Möhlenkamp, T., Moretto, L.G., Ocker, B., Pochodzalla, J., Raciti, G., Reito, S., Sann, H., Schüttauf, A., Seidel, W., Serfling, V., Trautmann, W., Trzcinski, A., Verde, G., Wörner, A., Zude, E., Zwieglinski, B., Z. Phys. A353, 197 (1995)

[17] Wirth, G., Brüchle, W., Brügger, M., Fan Worer, K., Funke, F., Kratz, J.V., Lerch, M., Trautmann, N., Phys.Lett. B177, 282 (1986)

[18] Wirth, G., Brüchle, W., Fan Wo, Sümmerer, K., Funke, F., Kratz, J.V., Trautmann, N., Oberacker, V.E., Z.Phys. A330, 87 (1988)

[19] Funke, F., Kratz, J.V., Trautmann, N., Wiehl, N., Wirth, G., Brüchle, W., Fan Wo, Sümmerer, K., Z.Phys., 340, 303 (1991)

[20] Wirth, G., Funke, F., Brüchle, W., Fan Wo, Kratz, J.V., Sümmerer, K., Trautmann, N., Phys. Lett. B253, 28 (1991)

[21] Piasecki, E., Pienkowski, L., Muchorowska, M., Tucholski, A., Czosnyka, T., Chbihi, A., Crema, E., Czarnacki, W., Galin, J., Guerreau, D., Iwanicki, J., Jacquet, D., Jahnke, U., Jastrzebski, J., Kisielinski, M., Kordyasz, A., Lewitowicz, M., Morjean, M., Pouthas, J., Phys. Lett. B377, 235 (1996)

[22] Broglia, R.A., and Winther, A., "Heavy Ion Reactions: Lectures Notes", 2nd Edition, Addison-Wesley, 1991, Redwood City, Calif. 
[23] Bonaccorso, A., Piccolo, G., Brink, D.M., Nucl.Phys. A441, 555 (1985)

[24] Bonaccorso, A., Brink, D.M., Lo Monaco, L., J.Phys.G: Nucl.Phys. 13, 1407(1987)

[25] Bonaccorso, A., Brink, D.M., Phys.Rev. C38, 1776 (1988)

[26] Bonaccorso, A., Brink, D.M., ibid. 43, 299 (1991)

[27] Bonaccorso, A., Brink, D.M., ibid. 44, 1559 (1991)

[28] Baur,G., Pauli,M., and Trautmann,D., Nucl.Phys.A224, 477 (1974);

Baur,G., and Trautmann,D., Phys.Rep.25 , 293(1976).

[29] Siemens, P.J., Bondorf, J., Gross, D.H.E., Dickmann, F., Phys. Lett. 36B, 24 (1971)

[30] Mermaz, M.C., Borrel, V., Guerreau, D., Galin, J., Gatty, B., Jacquet, D., Z. Phys. A324, 217 (1986)

[31] Breit, G., Ebel, M.E., Phys. Rev., 103, 679 (1956)

[32] von Oertzen, W., Phys.Lett. 151B, 95 (1985)

[33] Gupta, R.K., Maass, R., Scheid, W., Phys.Rev. C37, 1502 (1988)

[34] Broglia, R.A., Winther, A., Nucl. Phys. A182, 112 (1972)

[35] Islam, M.N., Siddiqua, N. and Harun-ar-Rashid, A.K.M., J. Phys. G20, 1471,1994

[36] Landau,L.D., and Lifshitz, E.M.,Quantum Mechanics, Pergamon Press, Oxford (1977), Chap. XVII, par. 127.

[37] Bertulani, C.A., Baur, G., Phys. Rep. 163, 299 (1988)

[38] Fermi, E., Z.Phys. 29, 315 (1924

[39] Weizsäcker, C.F., Z.Phys. 88, 612 (1934)

[40] Williams, E.J., Phys.Rev. 45, 729 (1934)

[41] Williams, E.J., K.Dan.Vidensk.Selsk.Mat.-Fys.Medd. 13 (1935) no.4 
[42] Justice, M.L., Ph.D. thesis, LBL report no. 31704, 1991, unpublished

[43] Veyssiére, A., Beil, H., Bergère, R., Carlos, P., Lepretre, A., Kernbath, K., Nucl.Phys. A199, 45 (1973)

[44] Caldwell, J.T., Dowdy, E.J., Berman, B.L., Alvarez, R.A., Meyer, P., Phys.Rev. C21, 1215 (1980)

[45] Aumann, T., Sümmerer,el, H., Blank, B., Brohm, T., Clerc, H.-G., Czajkowski, S., Donzaud, C., Grewe, A., Hanelt, E., Heinz, A., Irnich, H., de Jong, M., Junghans, A., Kratz, J.V., Magel, A., Münzenbckel, F., Pfützner, M., Piechaczek, A., Röhl, C., Scheidenberger, C., Schmidt, K.-H., Schwab, W., Steinhäuser, S., Trinder, W., Voss, B., Z. Phys., A352, 163 (1995)

[46] Rubehn, Th., Ph.D. thesis, University of Frankfurt (1995), unpublished

[47] Weber, Th., Heil, R.D., Kneissl, U., Wilke, W., Kihm, Th., Knöpfle, K.T., Emrich, H.J., Nucl. Phys. A510, 1 (1990)

[48] Pitthan, R., Buskirk,F.R., Houk, W.A., Moore, R.W., Phys. Rev. C21, 28 (1980)

[49] Winther, A., Alder, K., Nucl. Phys. A319, 518 (1979)

[50] Aleixo, A.N.F., Bertulani, C.A., Nucl. Phys. A505, 448 (1989)

[51] Zardi, F., Lenzi, S.M., Vitturi, A., Phys.Rev. C49, 1635 (1994) 


\begin{tabular}{lccc}
\hline \multicolumn{2}{c}{ State } & $\varepsilon_{i}(\mathrm{MeV})$ & $C_{1}\left(\mathrm{fm}^{-\frac{1}{2}}\right)$ \\
\hline Initial & $3 \mathrm{p}_{1 / 2}$ & -8.059 & 52.17 \\
Final & $3 \mathrm{~d}_{5 / 2}$ & -4.806 & 14.48 \\
\hline
\end{tabular}

Table : Initial and final neutron state parameters in ${ }^{197} \mathrm{Au}$ and ${ }^{239} \mathrm{U}$, respectively. 


\section{Figure captions}

Fig. 1. Dependence on the distance of closest approach of the calculated cross sections for in transfer to ground state (full symbols) and to unbound states (empty symbols) of ${ }^{239} \mathrm{U}$ in the reaction ${ }^{197} \mathrm{Au}\left({ }^{238} U,{ }^{239} U\right){ }^{196} \mathrm{Au}$ at three projectile energies $(8.1,24.3$ and $50 \mathrm{MeV} / \mathrm{u})$. The dependence on projectile energy of the latter cross section integrated over $D>25 \mathrm{fm}$ is presented in the inset.

Fig. 2. Calculated ${ }^{239} \mathrm{U}$ excitation energy distributions after $1 n$ transfer for three values of the distance of closest approach. $E_{\text {proj }}=24.3 \mathrm{MeV} / \mathrm{u}$. Each distribution has been normalized to one.

Fig. 3. The dependence on $\mathrm{D}$ of the cross section of 1 n transfer to unbound states of ${ }^{239} \mathrm{U}$. $\mathrm{E}_{\text {proj }}=24.3 \mathrm{MeV} / \mathrm{u}$. Three ranges of excitation energy, given in $\mathrm{MeV}$, are shown (for details - see text).

Fig. 4. Calculated spectra of virtual photons (left scale) and photoabsorption cross sections (right scale) for multipolarities E1 and E2 (isoscalar and isovector).

Fig. 5. Calculated excitation energy distributions of ${ }^{238} \mathrm{U}$ undergoing fission induced by electromagnetic field of multipolarity E1 (solid curve) and E2 isoscalar (dashed line) and isovector (dot-dashed line).

Fig. 6. The projectile energy dependence of the Coulomb fission cross sections $\sigma^{E 1}, \sigma^{E 2 s}, \sigma^{E 2 v}$ and their sum (for D $\geq 25 \mathrm{fm}$ ).

Fig. 7. Comparison of the dependence on $\mathrm{D}$ of the calculated cross sections for Coulomb fission and $\mathrm{In}$ transfer fission in the reaction $24.3 \mathrm{MeV} / \mathrm{u}$ ${ }^{238} \mathrm{U}+{ }^{197} \mathrm{Au}$. The latter one was obtained by multiplication of the transfer cross section by the fission probability $\mathrm{P}_{f}=0.22[45]$.

Fig. 8. Comparison between the experimental data and theoretical results for the $\mathrm{D}$ dependence of the fission cross sections. All the curves and experimental data are arbitrarily normalized at $D=18 \mathrm{fm}$. The latter concern the subbarrier 1n transfer [19] (full squares) and the deconvoluted results for CF [21] (full circles) supplemented by the statistical error bars. Theoretical curves pertain the $1 \mathrm{n}$ transfer in ${ }^{238} \mathrm{U}+{ }^{197} \mathrm{Au}$ reaction at $8.1 \mathrm{MeV} / \mathrm{u}$ (empty triangles) and $24.3 \mathrm{MeV} / \mathrm{u}$ (diamonds) as well as the CF in the same reaction (empty circles). One can see that the slope of the CF data for $\mathrm{D}>19 \mathrm{fm}$ is the same as that of the theoretical curve. 
Fig. 1

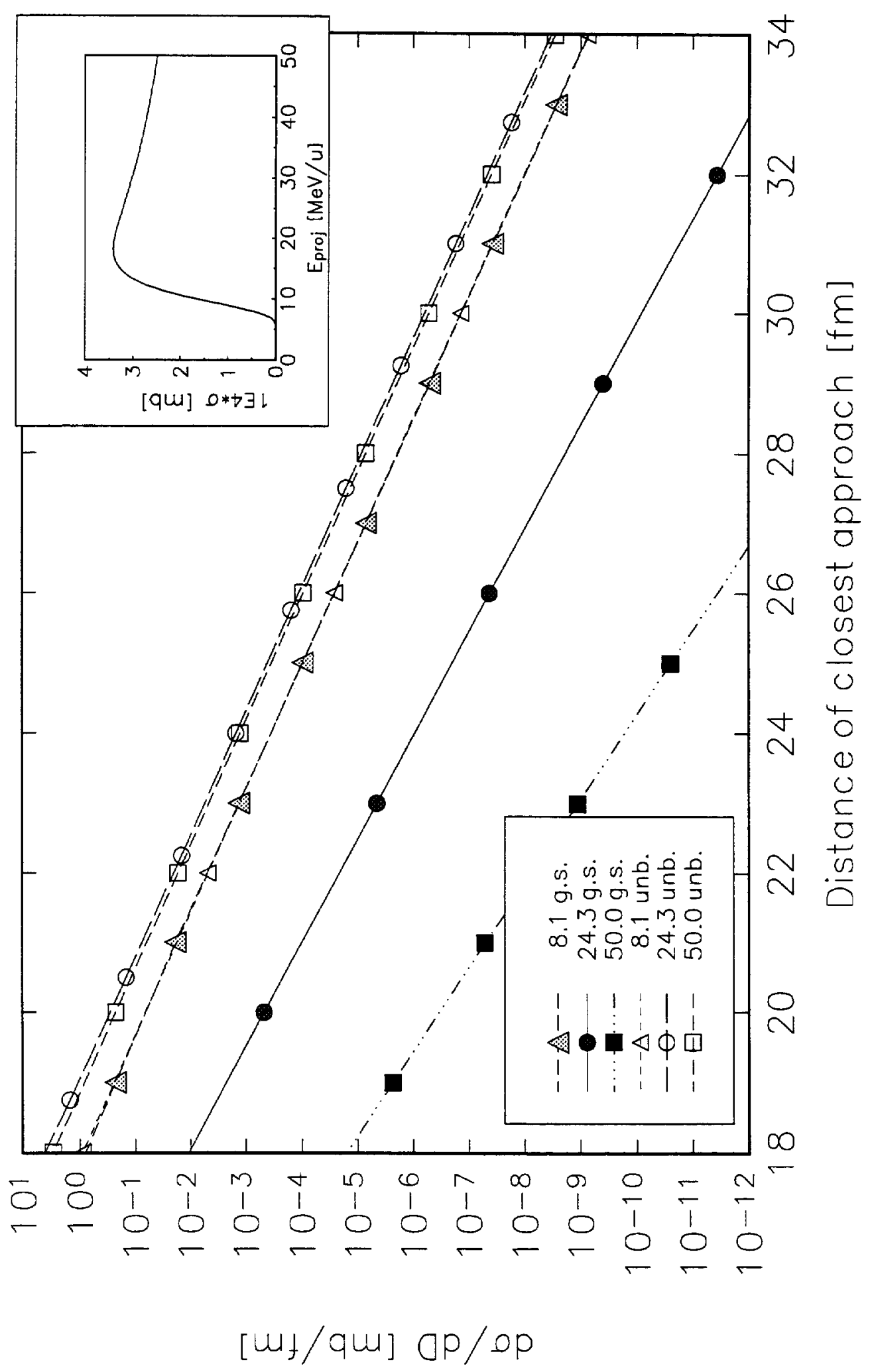


Fig. 2

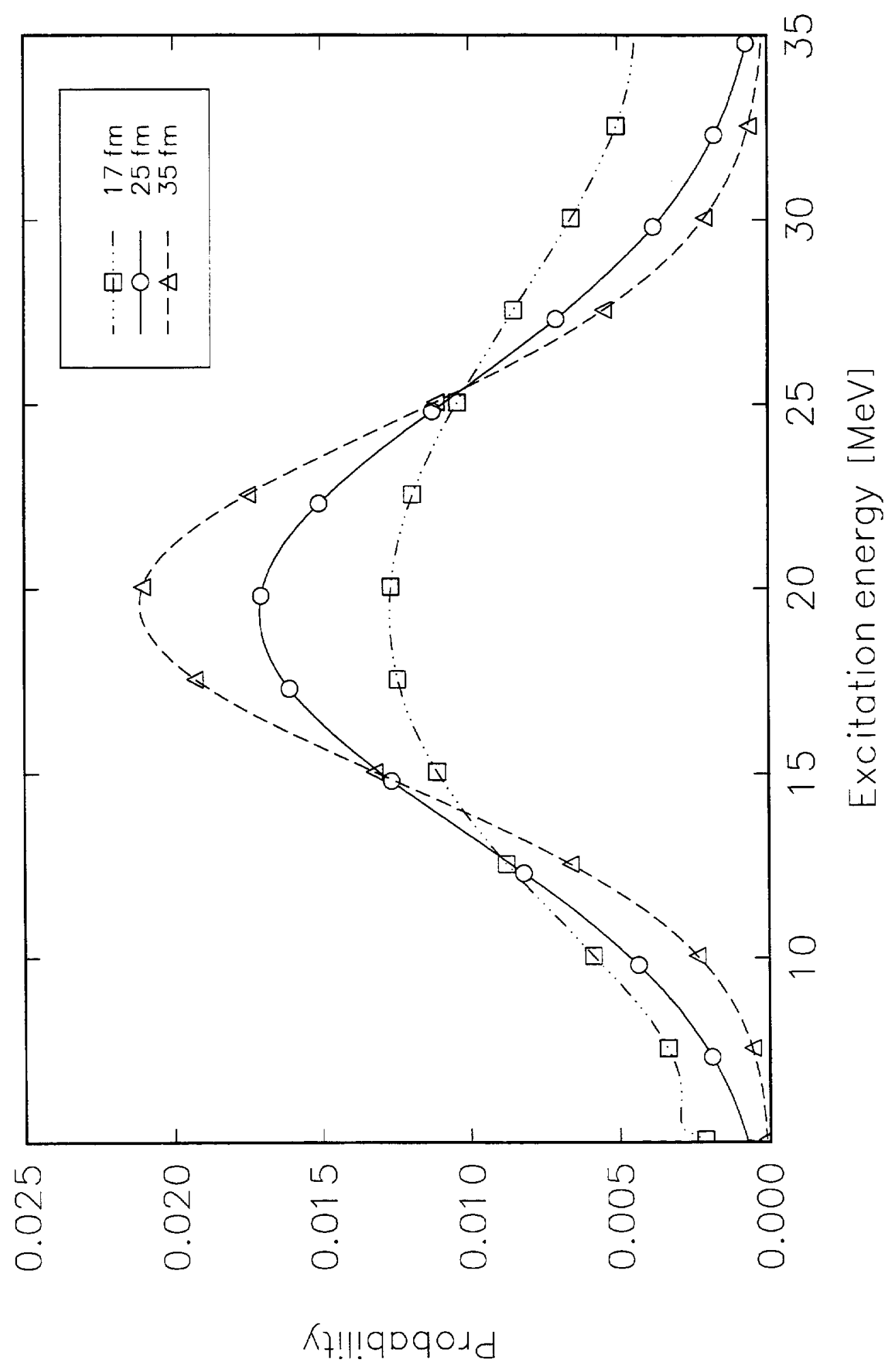


Fig. 3

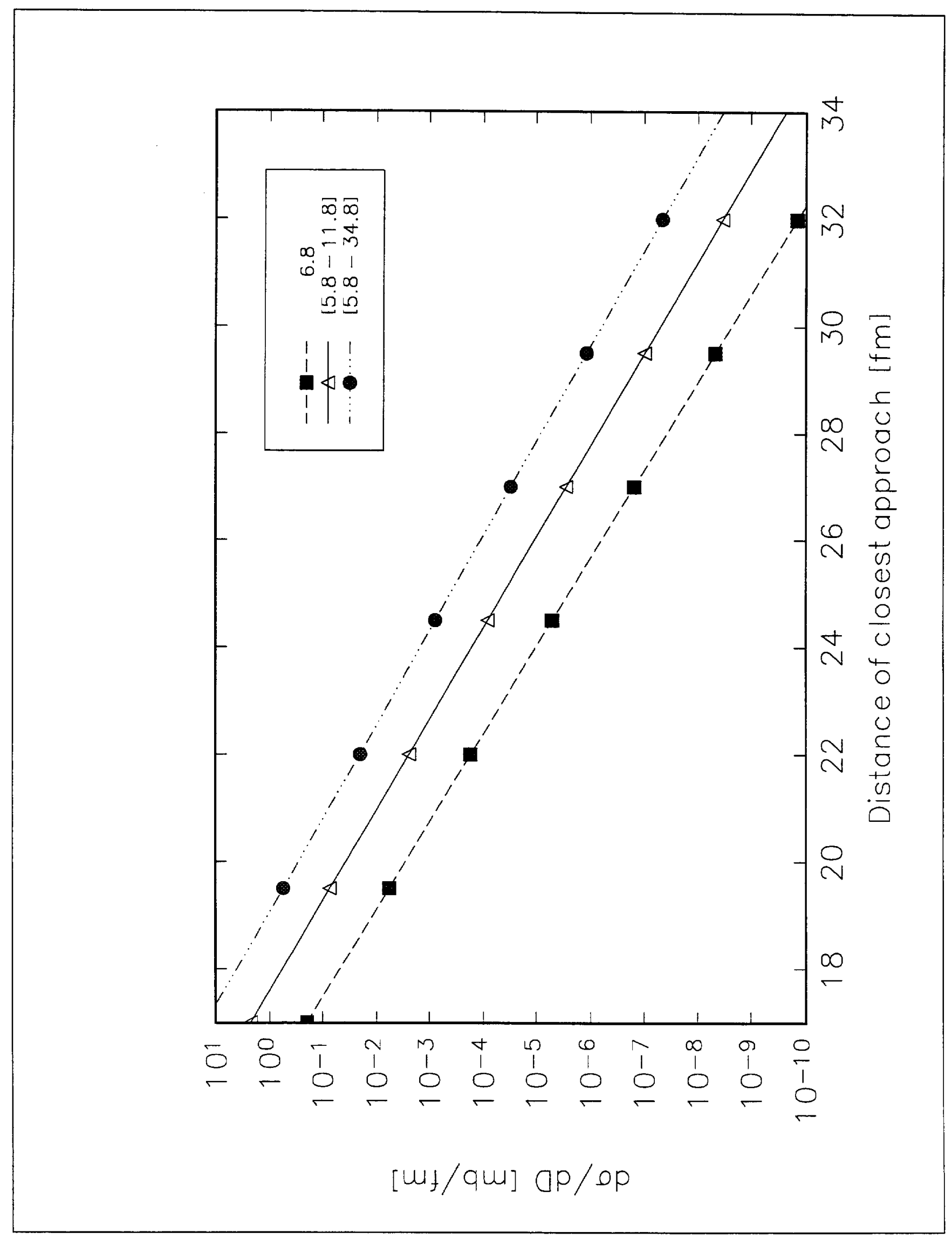


Fig. 4

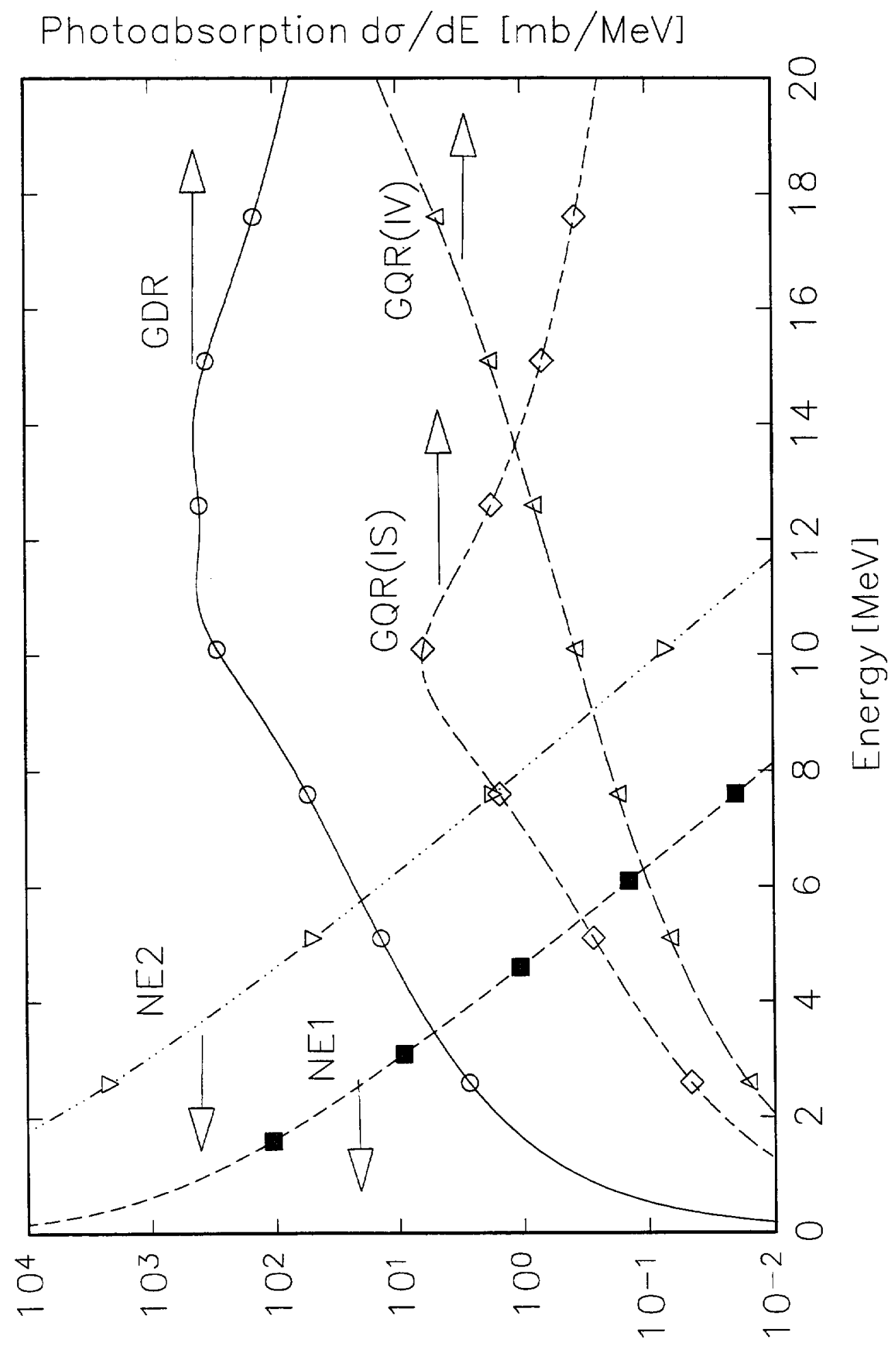

suofoud ionfl! to dəqunN 
Fig. 5

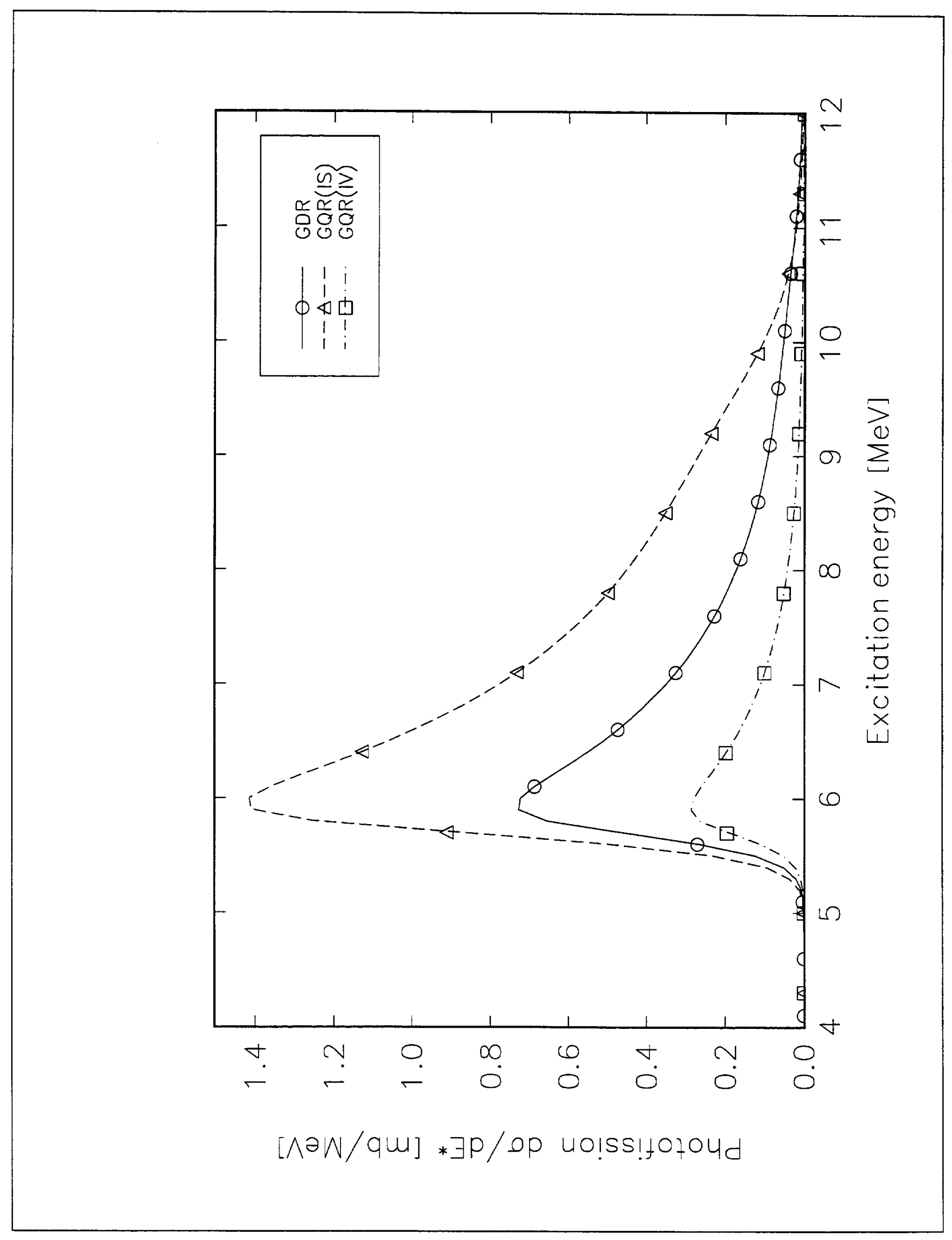




\section{Fig. 6}

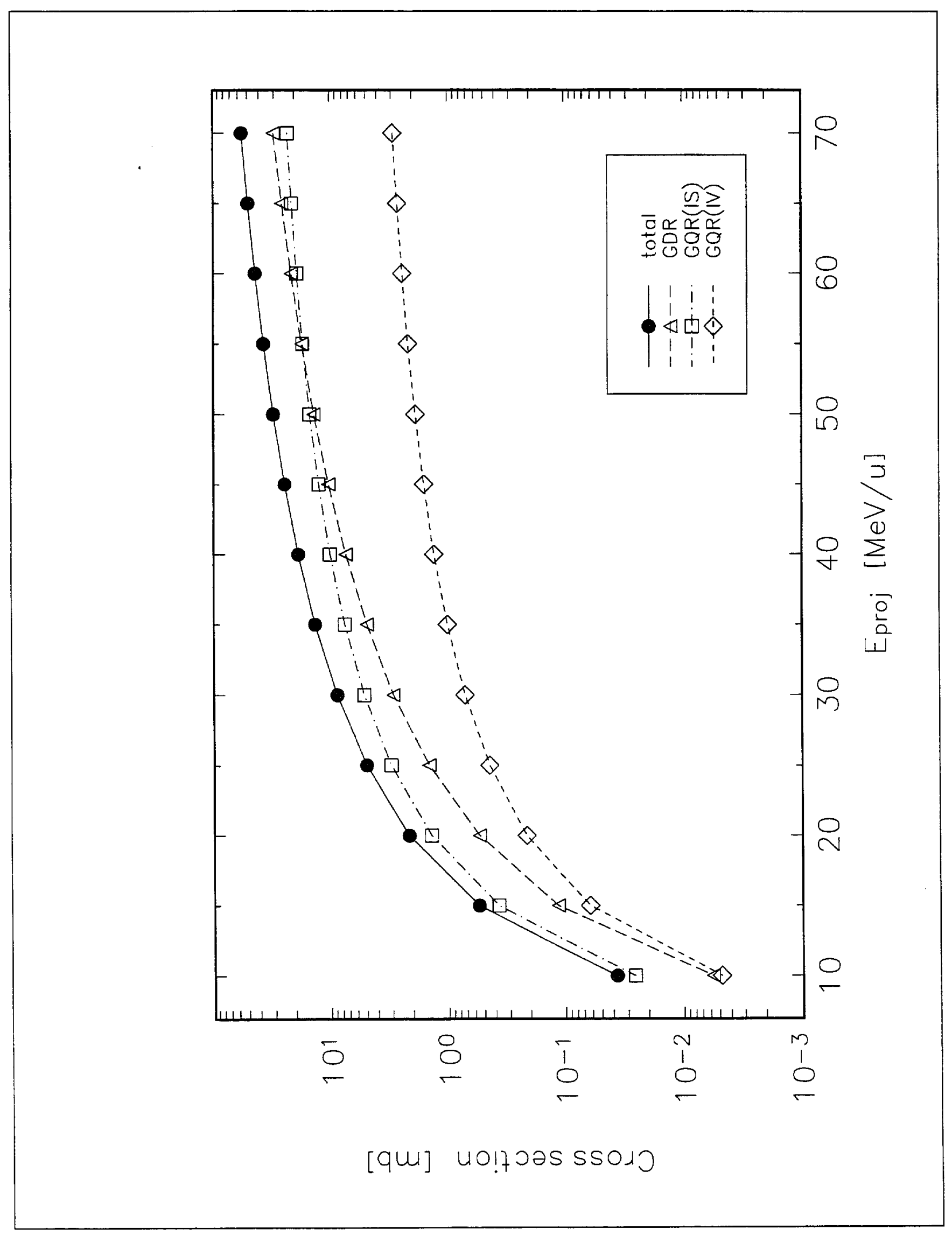


ing.7

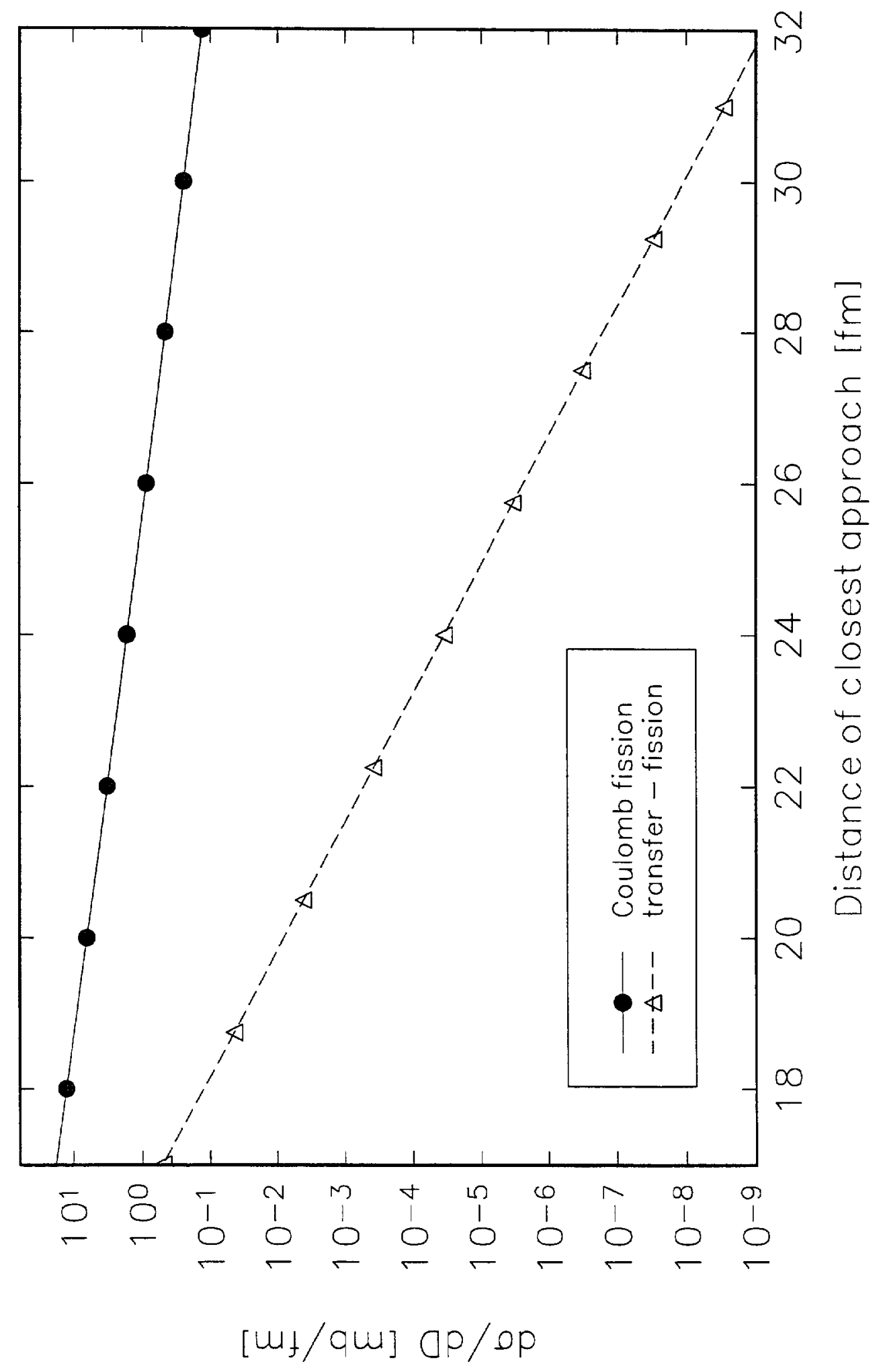


Fig. 8

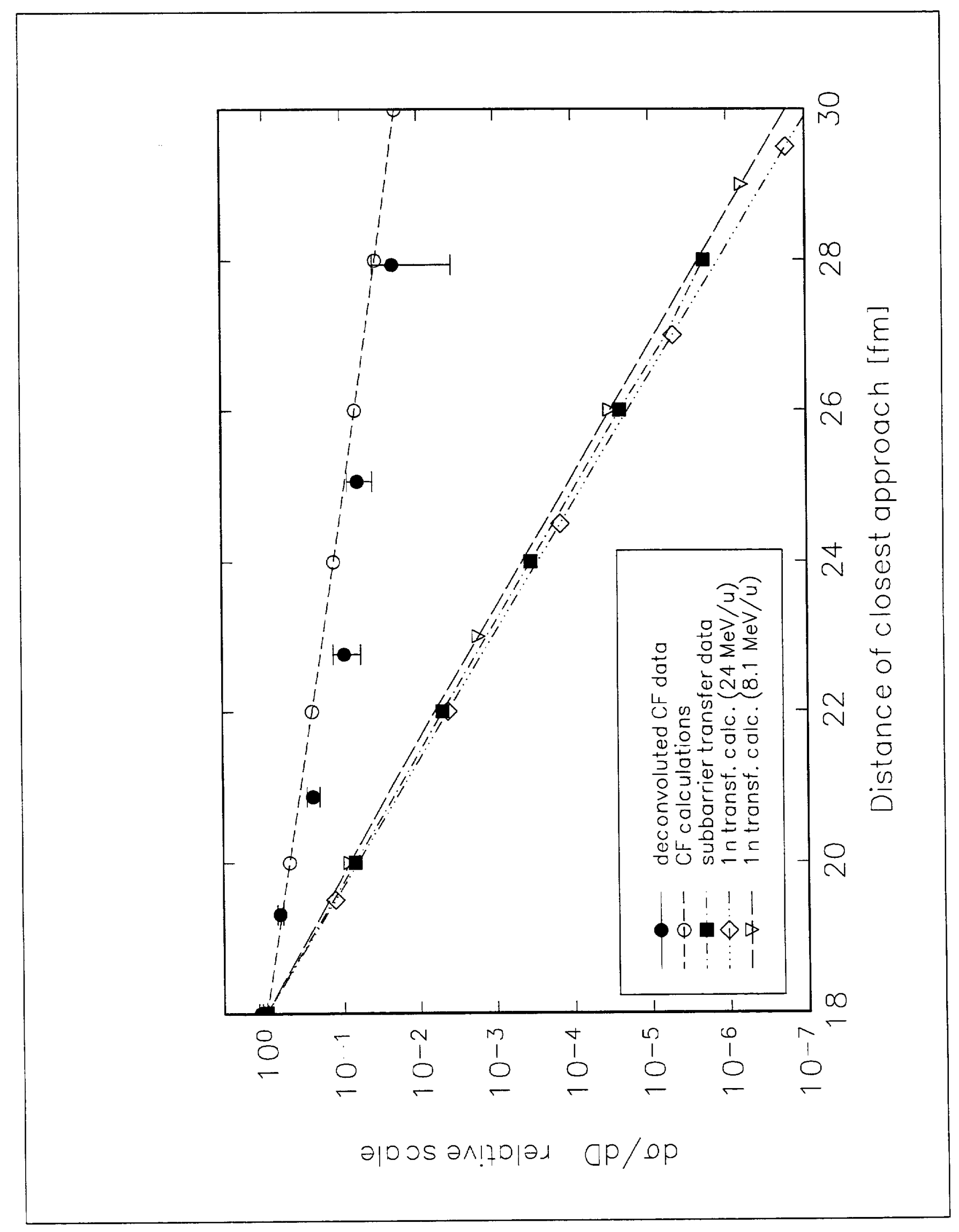




.

